01;02

\title{
Новый подход к вычислению потенциальной энергии взаимодействия двух атомов
}

\author{
(C) В.П. Кощеев ${ }^{1}$, Ю.Н. Штанов ${ }^{2}$ \\ ${ }^{1}$ Филиал „Стрела“ Московского авиационного института (Национального \\ исследовательского университета), Жуковский, Московская обл., Россия \\ ${ }^{2}$ Филиал Тюменского индустриального университета в г. Сургут, Сургут, \\ Россия \\ E-mail: koshcheev1@yandex.ru
}

Поступило в Редакцию 24 ноября 2017 г.

Фурье-компонента потенциальной энергии взаимодействия двух атомов представлена в виде многочлена четвертой степени от атомного формфактора. Численный расчет выполнен в приближении экранированного кулоновского потенциала. Показано, что учет принципа Паули приводит к появлению потенциального барьера и дополнительной области притяжения двух атомов. Показано, что данная модель качественно согласуется с результатами расчета с помощью теории функционала плотности.

DOI: 10.21883/PJTF.2018.13.46324.17133

В [1] была исследована проблема применения метода функционала плотности (см., например, [2]) к задаче вычисления потенциальной энергии взаимодействия атомов и ионов. В [3] был предложен альтернативный вариант решения этой задачи, в котором учет принципа Паули для одного из атомов привел к появлению потенциального барьера. Дальнейшее развитие подхода [3] представлено в настоящей работе.

Рассмотрим потенциальную энергию взаимодействия атома с зарядом ядра $Z_{1} e$ с атомом, заряд ядра которого равен $Z_{2} e$,

$$
U=\frac{Z_{1} Z_{2} e^{2}}{\left|\mathbf{r}_{1}-\mathbf{r}_{2}\right|}+\sum_{j_{i}=1}^{Z_{1}} \sum_{j_{2}=1}^{Z_{2}} \frac{e^{2}}{\left|\mathbf{r}_{1 j_{i}}-\mathbf{r}_{2 j_{2}}\right|}-\sum_{j_{2}=1}^{Z_{2}} \frac{Z_{1} e^{2}}{\left|\mathbf{r}_{1}-\mathbf{r}_{2 j_{2}}\right|}-\sum_{j_{1}=1}^{Z_{1}} \frac{Z_{2} e^{2}}{\left|\mathbf{r}_{2}-\mathbf{r}_{1 j_{1}}\right|}
$$


где $\mathbf{r}_{1}$ и $\mathbf{r}_{2}-$ векторы, определяющие положение ядер атомов; $\mathbf{r}_{1 j_{1}}=\mathbf{r}_{1}+\delta \mathbf{r}_{j 1}$ и $\mathbf{r}_{2 j_{2}}=\mathbf{r}_{2}+\delta \mathbf{r}_{j 2}-$ векторы, определяющие положение электрона $j_{1}$ первого атома и электрона $j_{2}$ второго атома.

Флуктуации потенциальной энергии взаимодействия (1) вызываются квантовыми флуктуациями, которые испытывают электроны. Усреднение по квантовым флуктуациям местоположения электронов будем осуществлять с помощью метода [4], который Бете использовал для вычисления атомного формфактора. Проведем усреднение (1) по квадратам модулей волновых функций атомов. Соответствующие средние будем обозначать $\langle\ldots\rangle_{e 1},\langle\ldots\rangle_{e 2}$.

Разложим потенциальную энергию взаимодействия (1) в интеграл Фурье

$$
\begin{aligned}
U= & \int \frac{d^{3} \mathbf{k}}{(2 \pi)^{3}}\left(\frac{4 \pi Z_{1} Z_{2} e^{2}}{k^{2}} \exp \left(i \mathbf{k}\left(\mathbf{r}_{1}-\mathbf{r}_{2}\right)\right)\right. \\
& +\frac{4 \pi e^{2}}{k^{2}} \sum_{j_{1}=1}^{Z_{1}} \sum_{j_{2}=1}^{Z_{2}} \exp \left(i \mathbf{k}\left(\mathbf{r}_{1 j_{1}}-\mathbf{r}_{2 j_{2}}\right)\right) \\
& \left.-\frac{4 \pi Z_{2} e^{2}}{k^{2}} \sum_{j_{1}=1}^{Z_{1}} \exp \left(i \mathbf{k}\left(\mathbf{r}_{2}-\mathbf{r}_{1 j_{1}}\right)\right)-\frac{4 \pi Z_{1} e^{2}}{k^{2}} \sum_{j_{2}=1}^{Z_{2}} \exp \left(i \mathbf{k}\left(\mathbf{r}_{1}-\mathbf{r}_{2 j_{2}}\right)\right)\right)
\end{aligned}
$$

Усредним (2) по квадрату модуля волновой функции первого атома

$$
\begin{aligned}
& \langle U\rangle_{e 1}=\int \frac{d^{3} \mathbf{k}}{(2 \pi)^{3}}\left(\frac{4 \pi Z_{1} Z_{2} e^{2}}{k^{2}} \exp \left(i \mathbf{k}\left(\mathbf{r}_{1}-\mathbf{r}_{2}\right)\right)\right. \\
& +\frac{4 \pi e^{2}}{k^{2}}\left\langle\sum_{j_{1}=1}^{Z_{1}} \sum_{j_{2}=1}^{Z_{2}} \exp \left(i \mathbf{k}\left(r_{1 j_{1}}-\mathbf{r}_{2 j_{2}}\right)\right)\right\rangle_{e 1} \\
& \left.-\frac{4 \pi Z_{2} e^{2}}{k^{2}}\left\langle\sum_{j_{1}=1}^{Z_{1}} \exp \left(i \mathbf{k}\left(\mathbf{r}_{2}-\mathbf{r}_{1 j_{1}}\right)\right)\right\rangle_{e l}-\frac{4 \pi Z_{1} e^{2}}{k^{2}} \sum_{j_{2}=1}^{Z_{2}} \exp \left(i \mathbf{k}\left(\mathbf{r}_{1}-\mathbf{r}_{2 j_{2}}\right)\right)\right) \\
& \quad\left\langle\sum_{j_{1}=1}^{Z_{1}} \sum_{j_{2}=1}^{Z_{2}} \exp \left(i \mathbf{k}\left(\mathbf{r}_{1 j_{1}}-\mathbf{r}_{2 j_{2}}\right)\right)\right\rangle_{e 1}=F_{1}(k) \sum_{j_{2}=1}^{Z_{2}} \exp \left(i \mathbf{k}\left(\mathbf{r}_{1}-\mathbf{r}_{2 j_{2}}\right)\right)
\end{aligned}
$$

Письма в ЖТФ, 2018, том 44, вып. 13 


$$
\left\langle\sum_{j_{1}=1}^{Z_{1}} \exp \left(i \mathbf{k}\left(\mathbf{r}_{2}-\mathbf{r}_{1 j_{1}}\right)\right)\right\rangle_{e 1}=F_{1}(k) \exp \left(i \mathbf{k}\left(\mathbf{r}_{1}-\mathbf{r}_{2}\right)\right),
$$

где $F_{1}(k)$ - атомный формфактор, $F_{1}(0)=Z_{1}$.

Проведем усреднение $\langle U\rangle_{e 1}$ по квадрату модуля волновой функции второго атома

$$
\begin{array}{r}
\langle U\rangle_{e 1, e 2}=\int \frac{d^{3} \mathbf{k}}{(2 \pi)^{3}}\left(\frac{4 \pi Z_{2} e^{2}}{k^{2}}\left(Z_{1}-F_{1}(k)\right) \exp \left(i \mathbf{k}\left(\mathbf{r}_{1}-\mathbf{r}_{2}\right)\right)\right. \\
\left.\quad-\frac{4 \pi e^{2}}{k^{2}}\left(Z_{1}-F_{1}(k)\right)\left\langle\sum_{j_{2}=1}^{Z_{2}} \exp \left(i \mathbf{k}\left(\mathbf{r}_{1}-\mathbf{r}_{2 j_{2}}\right)\right)\right\rangle_{e 2}\right), \\
\left\langle\sum_{j_{2}=1}^{Z_{2}} \exp \left(i \mathbf{k}\left(\mathbf{r}_{1}-\mathbf{r}_{2 j_{2}}\right)\right)\right\rangle_{e 2}=F_{2}(k) \exp \left(i \mathbf{k}\left(\mathbf{r}_{1}-\mathbf{r}_{2}\right)\right) .
\end{array}
$$

Таким образом, потенциальная энергия взаимодействия двух атомов, усредненная по квадратам модулей волновых функций, имеет вид

$$
\langle U\rangle_{e 1, e 2}=\int \frac{d^{3} \mathbf{k}}{(2 \pi)^{3}} \frac{4 \pi e^{2}}{k^{2}}\left(Z_{1}-F_{1}(k)\right)\left(Z_{2}-F_{2}(k)\right) \exp \left(i \mathbf{k}\left(\mathbf{r}_{1}-\mathbf{r}_{2}\right)\right),
$$

где $U(k)=\frac{4 \pi e^{2}}{k^{2}}\left[Z_{1}-F_{1}(k)\right]\left[Z_{2}-F_{2}(k)\right]$ - фурье-компонента потенциальной энергии взаимодействия двух атомов.

Аналогично тому как это делается в кинетической теории [5], добавим к выражению для фурье-компоненты потенциальной энергии взаимодействия двух атомов множитель $(1-F(k) / Z)$, с помощью которого будем учитывать принцип Паули. Величина $F(k) / Z$ является фурье-компонентой плотности распределения электронов, которая нормирована на единицу. Ожидается, что сомножитель $(1-F(k) / Z)$ будет действовать аналогично функции распределения Ферми-Дирака, с помощью которой учитывают принцип Паули в системах частиц с полуцелым спином [4]. В [3] принцип Паули был учтен для одного из двух атомов с помощью дополнительного сомножителя

$$
U(k)=\frac{4 \pi e^{2}}{k^{2}}\left[Z_{1}-F_{1}(k)\right]\left[Z_{2}-F_{2}(k)\right]\left[1-\frac{F_{2}(k)}{Z_{2}}\right] .
$$

Письма в ЖТФ, 2018, том 44, вып. 13 
В настоящей работе учтем принцип Паули для обоих атомов с помощью двух дополнительных сомножителей

$$
U(k)=\frac{4 \pi Z_{1} Z_{2} e^{2}}{k^{2}}\left[1-\frac{F_{1}(k)}{Z_{1}}\right]^{2}\left[1-\frac{F_{2}(k)}{Z_{2}}\right]^{2} .
$$

Численный расчет выполним для случая $Z_{1}=Z_{2}=Z$,

$$
U_{n}(k)=\frac{4 \pi(Z e)^{2}}{k^{2}}\left[1-\frac{F(k)}{Z}\right]^{n} .
$$

Видно, что формула (8) при различных значениях целого числа $n=0,1,2,3,4$ описывает фурье-компоненту потенциальной энергии взаимодействия:

a) двух одинаковых атомных ядер при $n=0$;

b) атомного ядра и атома при $n=1$;

c) двух атомов без учета принципа Паули при $n=2$;

d) двух атомов с учетом принципа Паули для одного из них при $n=3$;

е) двух атомов с учетом принципа Паули для обоих атомов при $n=4$.

Атомный формфактор в приближении экранированного кулоновского потенциала запишем в виде

$$
F(k)=\frac{Z \mu^{2}}{\mu^{2}+k^{2}}
$$

где $\mu=1 / a, a$ - длина экранирования [4].

С помощью теории вычетов (см., например, [6]) по формулам (8) и (9) вычислим потенциальную энергию взаимодействия двух атомов

$$
U_{n}(r)=\frac{(Z e)^{2}}{r} \frac{2}{(n-1) !} \operatorname{Im}\left[i \frac{d^{(n-1)}}{d k^{(n-1)}}\left(\frac{k^{2 n-1} \exp [i k r]}{(k+i \mu)^{n}}\right)\right]_{k=i \mu},
$$

где $r=\left|\mathbf{r}_{1}-\mathbf{r}_{2}\right|, i=\sqrt{-1}, \operatorname{Im} z-$ мнимая часть комплексного числа $z, n !=n(n-1)$ !

Результат вычисления по формуле (10) для $n=0.1 .2 .3$ представлен в [3], а при $n=4$ имеет вид

$$
U_{4}(r)=\frac{(Z e)^{2}}{48 r}\left[-(\mu r)^{3}+15(\mu r)^{2}-57 \mu r+48\right] \exp (-\mu r) .
$$

Письма в ЖТФ, 2018, том 44, вып. 13 


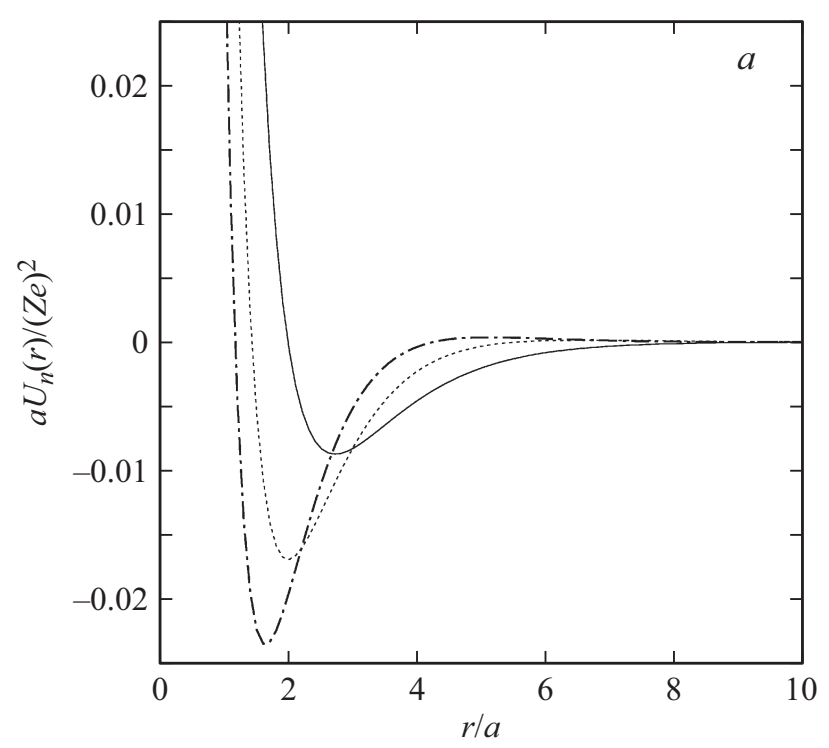

Рис. 1. Потенциальная энергия взаимодействия двух атомов без учета принципа Паули $U_{2}(r)$ (сплошная линия), с учетом принципа Паули для одного атома $U_{3}(r)$ (пунктирная линия) и с учетом принципа Паули для двух атомов $U_{4}(r)$ (штрихпунктирная линия) в зависимости от расстояния между ними.

Формула (11) описывает потенциальную энергию взаимодействия с учетом принципа Паули для двух атомов.

С помощью формулы (10) построим потенциальную энергию взаимодействия двух атомов без учета принципа Паули

$$
U_{2}(r)=\frac{(Z e)^{2}}{2 r}(2-\mu r) \exp (-\mu r) .
$$

На рис. 1, $a$ представлены графики потенциальной энергии взаимодействия с учетом $U_{4}(r)$ и без учета $U_{2}(r)$ принципа Паули для двух атомов. Учет принципа Паули для двух атомов приводит к появлению как потенциального барьера, так и дополнительной области притяжения двух атомов, что в увеличенном масштабе представлено на рис. $1, b$ и $c$ соответственно. На рис. $1, a-c$ показаны также графики потенциальной энергии взаимодействия для двух атомов с учетом принципа Паули для

Письма в ЖТФ, 2018, том 44, вып. 13 

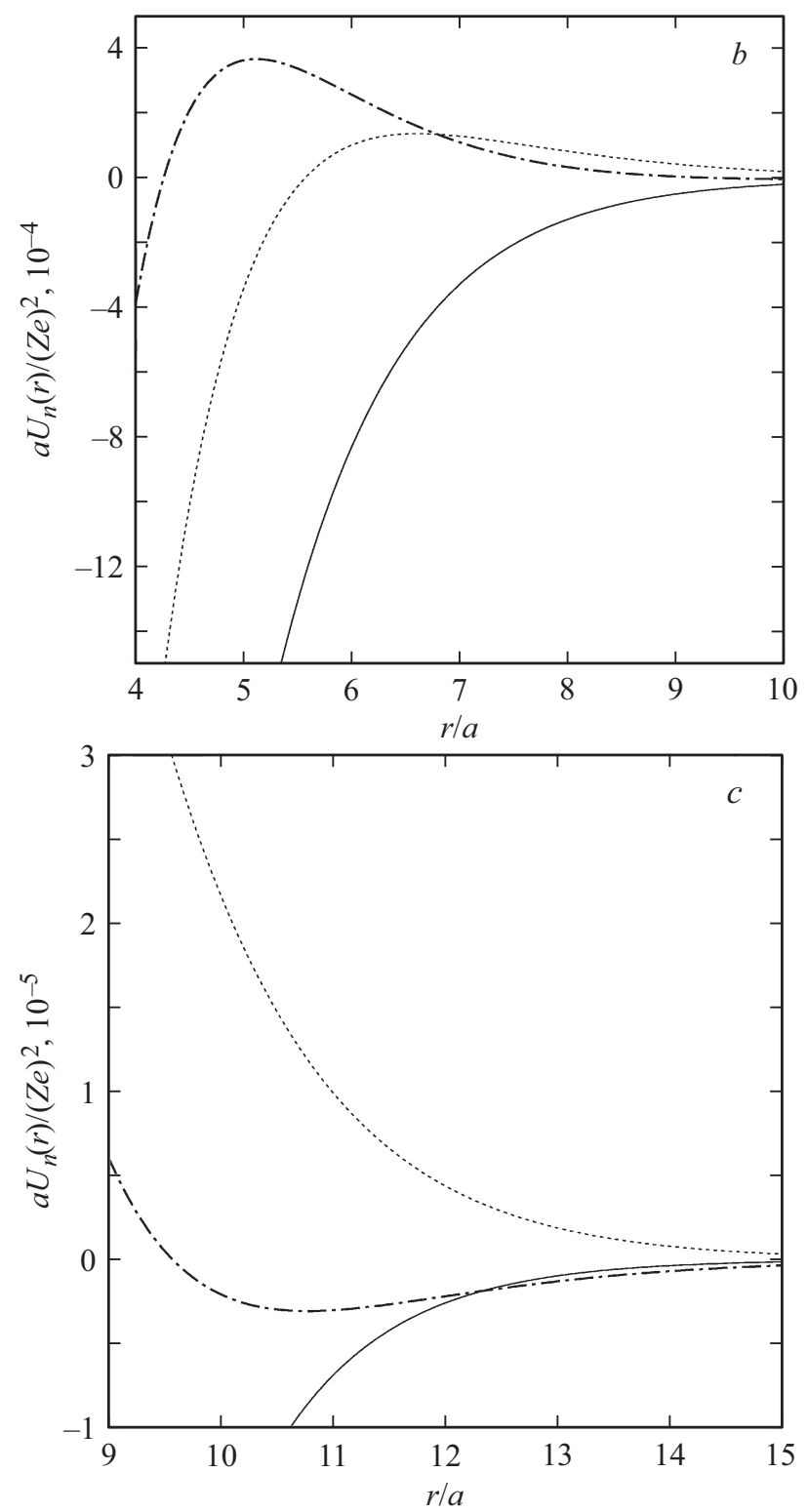

Рис. 1 (продолжение).

3 Письма в ЖТФ, 2018, том 44, вып. 13 


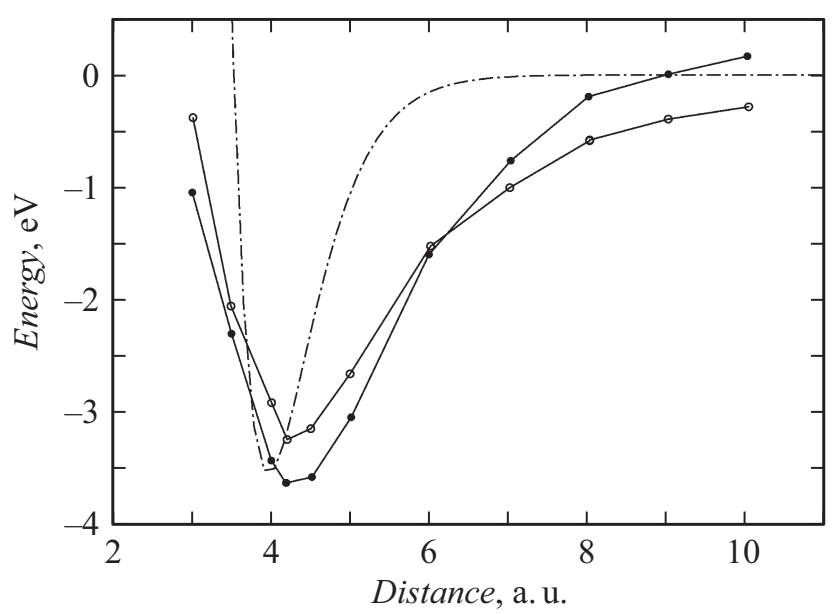

Рис. 2. Зависимость величины энергии связи в димере $\mathrm{Si}_{2}$ (на атом) от расстояния между атомами (данные работы [7]): темные точки — расчет по методу [7], светлые - расчет по методу Кона-Шэма (пакет FHI96md) (1 a. u. длины равняется $0.529 \AA)$. Результаты нашего расчета $U_{4}(r)$ были увеличены в 80 раз и представлены штрихпунктирной линией.

одного атома [3]

$$
U_{3}(r)=\frac{(Z e)^{2}}{8 r}\left((\mu r)^{2}-7 \mu r+8\right) \exp (-\mu r) .
$$

Видно, что дополнительная область притяжения отсутствует, а положение и высота потенциального барьера отличаются от полученных в случае $U_{4}(r)$. На рис. 2 представлено сравнение результатов расчета с данными, полученными в работе [7]. Для атома кремния длина экранирования $a=0.194 \AA$, a $\left(Z e^{2} / a\right) \approx 14.6 \mathrm{keV}$. Данная модель лишь качественно согласуется с результатами расчета с помощью теории функционала плотности, так как глубина потенциальной ямы

$$
U_{4}\left(r=r_{\min } \approx 3.95 \text { a. u. }\right) \approx 0.044 \mathrm{eV},
$$

что в 80 раз меньше, чем в [7]. Следует ожидать, что более реалистичные модели электронной плотности (см., например, [8]) позволят 
получить количественное согласие нового подхода к вычислению потенциальнойэнергии взаимодействия двух атомов с результатами расчета с помощью теории функционала плотности.

\section{Список литературы}

[1] Medvedev M. G., Bushmarinov I.S., Sun J., Perdew J.P., Lyssenko K.A. // Science. 2017. V. 355. N 6320. P. 49-52.

[2] Сарры А.М., Сарры М.Ф. // ФТТ. 2012. Т. 54. В. 6. С. 1237-1243.

[3] Панина Т.А., Кощеев В.П., Моргун Д.А., Сабин Н.В. // Изв. РАН. Сер. физ. 2010. T. 74. № 2. C. 180-183.

[4] Бете Г. Квантовая механика. М.: Мир, 1965. 333 с.

[5] Лифшии Е.М., Питаевский Л.П. Физическая кинетика. М.: Наука, 1979. $528 \mathrm{c}$.

[6] Свешников А.Г., Тихонов А.Н. Теория функций комплексной переменной. М.: Наука, 1974. $319 \mathrm{c}$.

[7] Заводинский В.Г., Горкуша О.А. // ФТТ. 2014. Т. 56. В. 11. С. 2253-2258.

[8] Kirkland E.J. Advanced computing in electron microscopy. Springer Science \& Business Media, 2010. 293 p.

$3^{*}$ Письма в ЖТФ, 2018, том 44, вып. 13 